\title{
Vibrational Relaxation Times of Diatomic Molecules and Rocket Performance*†
}

\author{
H. C. Penny $\ddagger$ and Henry Aroeste \\ Guggenheim Jet Propulsion Center, California Institute of Technology, Pasadena, California
}

(Received October 11, 1954)

\begin{abstract}
Computations are presented for the variation of vibrational excitation probabilities with temperature for mixtures of $\mathrm{O}_{2}$ and $\mathrm{N}_{2}$, and of $\mathrm{HF}$ and $\mathrm{H}_{2}$. The values of six different excitation probabilities in each mixture are reported for temperatures up to $3000^{\circ} \mathrm{K}$. For each of the probabilities there is a lower temperature limit given, below which the theory of Schwartz, Slawsky, and Herzfeld, which is used here, is not applicable. Using the results for $\mathrm{H}_{2}-\mathrm{HF}$ mixtures, we present some considerations which suggest that the expansion through the de Laval nozzle for representative $\mathrm{H}_{2}-\mathrm{F}_{2}$ rocket motors may be taken as vibrational nearequilibrium flow.
\end{abstract}

\section{CALCULATION OF VIBRATIONAL EXCITATION PROBABILITIES}

$\mathrm{F}$ R the computation on vibrational excitation probabilities we have used the method described by Schwartz, Slawsky and Herzfeld. ${ }^{1}$ For the mixture of molecules of type $a$ and $b$, we may define the probabilities $P_{0 \rightarrow 0}(a, a), P_{0 \rightarrow 0}(b, b), P_{0 \rightarrow 0}(a, b), P_{0 \rightarrow 0}(b, a), P_{0 \rightarrow 1}(a, b)$, $P_{0 \rightarrow 1}(b, a)$. In each of these cases, the molecule, the symbol of which appears first in the parenthesis, was

* Supported by contract with the Office of Naval Research and the Office of Ordnance Research.

$\uparrow$ Presented at the 126th National Meeting of the American Chemical Society, New York, New York, September, 1954.

$\$$ This article uses, in part, the results of a thesis submitted by H. C. Penny, Lieutenant, U. S. Navy, in partial fulfillment of requirements for the degree of Aeronautical Engineer, California Institute of Technology, June 1954.

${ }^{1}$ Schwartz, Slawsky, and Herzfeld, J. Chem. Phys. 20, 1591 (1952); R. Schwartz and K. Herzfeld, J. Chem. Phys, 22, 8768 (1954). We thank Dr. Schwartz and Professor Herzfeld for sending us a preprint of the latter paper. initially in the first excited vibrational state and finally in the ground, or zero, vibrational state. The subscript designates the behavior of the vibrational quantum number of the molecule, the symbol of which appears second in the parenthesis. Each of these probabilities has been tabulated and graphed as functions of temperature for both the $\mathrm{O}_{2}-\mathrm{N}_{2}$ and the $\mathrm{HF}-\mathrm{H}_{2}$ gaseous mixtures.

The probability $P_{0 \rightarrow 1}(b, a)$ may be obtained simply from the relation

$$
P_{0 \rightarrow 1}(b, a)=P_{0 \rightarrow 1}(a, b) \exp \left[-h\left(\nu_{a}-\nu_{b}\right) / k T\right] .
$$

The others are determined independently. Summarizing the results of Schwartz et al. with slight modification we write for the probabilities,

$$
\begin{aligned}
P & =C P^{\prime}, \\
P^{\prime} & =1.18\left[8 \pi^{2} \mu \Delta E / \alpha^{* 2} h^{2}\right]^{2} \sigma_{1}^{\frac{1}{2}} \exp (-\sigma) \prod_{i} V^{2}(i), \\
C & =0.45\left[\left(1+\sigma_{1} k T / 3 \epsilon\right)^{\frac{1}{2}}+1\right]^{\frac{1}{3}} \exp (-\epsilon / k T),
\end{aligned}
$$




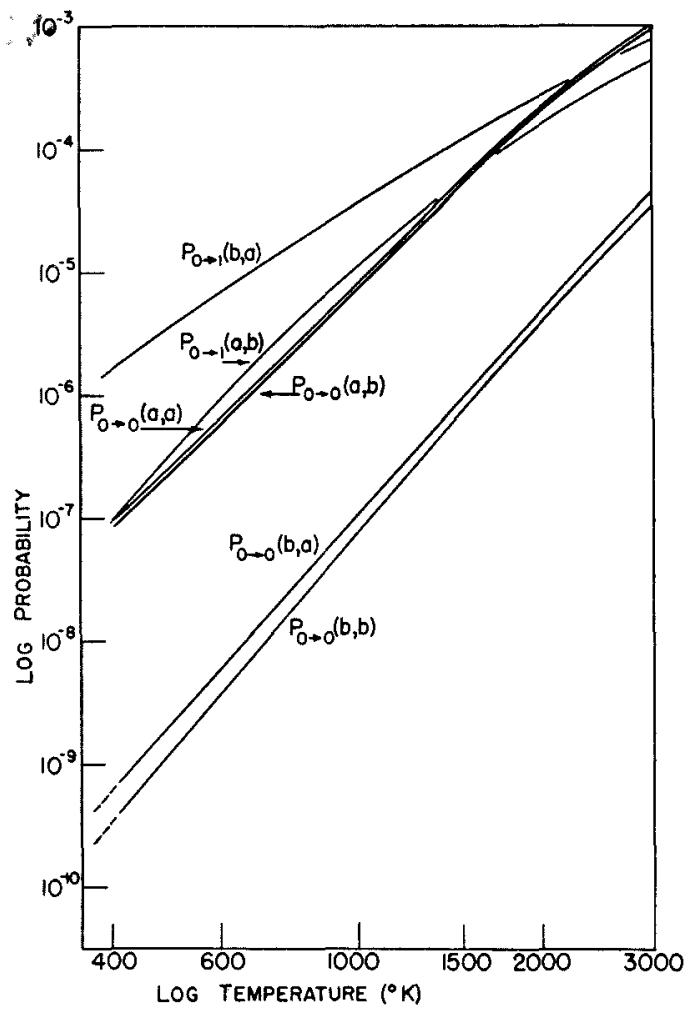

FIG. 1. Variation of probabilities with temperature $\left(a=\mathrm{O}_{2}\right.$ $b=\mathrm{N}_{2}$ ). Limit temperatures, below which the theory becomes inapplicable, correspond to the junction between the solid and dotted portions of the curves.

where

$$
\begin{aligned}
\sigma_{1} & =3 \mu v_{0}^{* 2} / 2 k T, \\
\sigma & =\sigma_{1} \pm \Delta E / 2 k T, \\
v_{0}^{*} & =\left[4 \pi^{2} k T \Delta E / \alpha^{*} h \mu\right]^{\frac{1}{3}} \\
& \mp(\Delta E / 2 \mu)\left[4 \pi^{2} k T \Delta E / \alpha^{*} h \mu\right]^{-\frac{1}{3}}+\cdots .
\end{aligned}
$$

Here $\mu$ is the reduced mass of the two colliding molecules, $\Delta E$ represents the amount of energy exchanged between vibration and translation, $\epsilon$ and $r_{0}$ are the usual constants in the Lennard-Jones interaction potential, and $V(i)$ is the perturbation integral over harmonic oscillator wave functions. For $\alpha^{*}$ we use the approximate relation $\alpha^{*}=17.5 / r_{0}$. In the case of HF, where no sufficient data is available, the collision diameter, $\boldsymbol{r}_{0}$, was estimated by plotting $r_{0}$ as a function of the electronegativity of the halogen atoms. In this manner it is

TABLE I. Lower temperature limits for which the probabilities can be calculated from the theory of Schwartz and Herzfeld.

\begin{tabular}{lcc}
\hline & \multicolumn{2}{c}{ Temperature ${ }^{\circ} \mathrm{K}$} \\
Probability & $\mathrm{O}_{2}-\mathrm{N}_{2}$ & $\mathrm{HF}-\mathrm{H}_{2}$ \\
\hline$P_{0 \rightarrow 0}(a, a)$ & 326 & 1390 \\
$P_{0 \rightarrow 0}(b, b)$ & 405 & 2700 \\
$P_{0 \rightarrow 0}(a, b)$ & 330 & 2380 \\
$P_{0 \rightarrow 0}(b, a)$ & 403 & 2440 \\
$P_{0 \rightarrow 1}(a, b)$ & 231 & 592 \\
$P_{0 \rightarrow 1}(b, a)$ & 231 & 592 \\
\hline
\end{tabular}

seen that the value of $r_{0}$ for $\mathrm{HF}$ is, approximately, $1.7 \mathrm{~A}$.

The condition

$$
\mu v_{0} * 2 / 2>\Delta E
$$

will not hold below a certain temperature for each of the probabilities. We need inequality (8) to write the expansion,

$$
v_{f}^{*}=v_{0}^{*}+\Delta E / \mu v_{0}^{*}-(\Delta E)^{2} / 2 \mu^{2} v_{0}^{* 3}+\cdots
$$

$\mathrm{It}$ is not necessary that

$$
\mu v_{0}^{* 2} / 2 \gg \Delta E,
$$

although the larger the inequality, the better the convergence. Schwartz and Herzfeld, in the appendix of their paper, have given a more complex method of computation which applies in the region of or below the limit temperatures. We have indicated these limit temperatures in Table I.

The above relationships have been used to compute the values of the probabilities listed in Table II which are shown graphically in Figs. 1 and 2. Using log scales for both the probabilities and the temperatures, the curves are seen to approximate straight lines except at the higher temperatures.

\section{APPLICATION TO ADIABATIC EXPANSION THROUGH DE LAVAL NOZZLES}

We now present brief considerations which enable us to determine whether or not the expansion through the

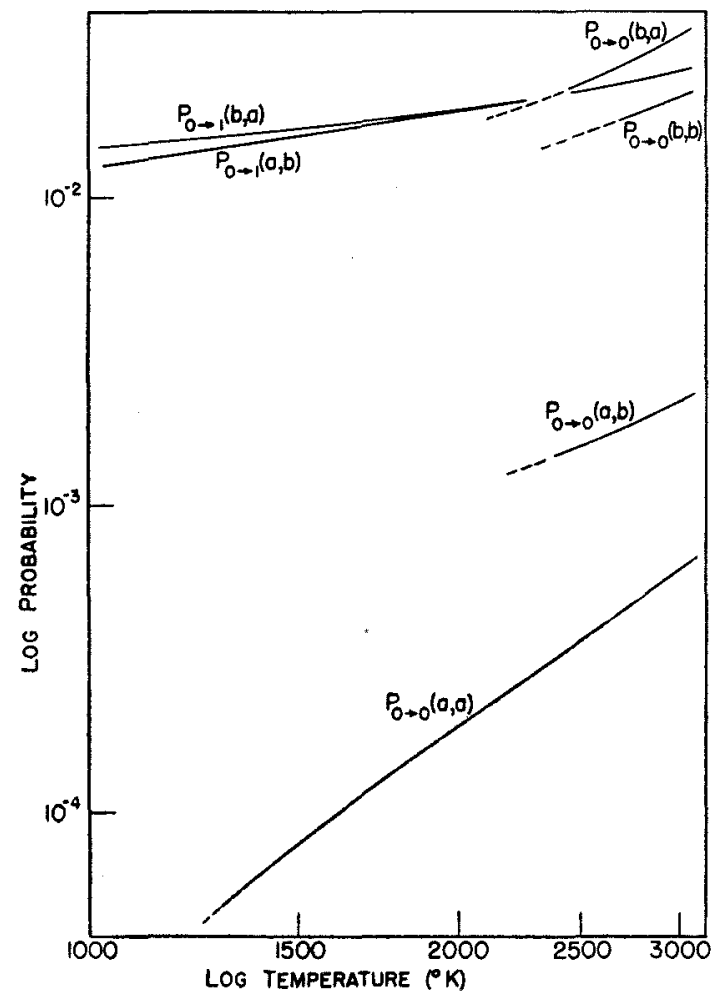

FIG. 2. Variation of probabilities with temperature $(a=\mathrm{HF}$, $b=\mathrm{H}_{2}$ ). Limit temperatures, below which the theory becomes inapplicable, correspond to the junction between the solid and dotted portions of the curves. 
TABLE II. Variation of probabilities with temperature.

\begin{tabular}{|c|c|c|c|c|c|c|}
\hline${ }^{\circ} \mathrm{K}$ & $P_{0 \rightarrow 0}(a, a)$ & $P_{0 \rightarrow 0}(b, b)$ & $P_{0 \rightarrow 0}(a, b)$ & $P_{0 \rightarrow 0}(b, a)$ & $P_{0 \rightarrow 1}(a, b)$ & $P_{0 \rightarrow 1}(b, a)$ \\
\hline \multicolumn{7}{|c|}{$\mathrm{O}_{2}-\mathrm{N}_{2}$} \\
\hline 400 & $8.8 \times 10^{-8}$ & $3.3 \times 10^{-10}$ & $9.0 \times 10^{-8}$ & $7.1 \times 10^{-10}$ & $9.5 \times 10^{-8}$ & $1.6 \times 10^{-6}$ \\
\hline 600 & $6.8 \times 10^{-7}$ & $3.6 \times 10^{-9}$ & $6.1 \times 10^{-7}$ & $5.7 \times 10^{-9}$ & $1.0 \times 10^{-6}$ & $6.9 \times 10^{-6}$ \\
\hline 1000 & $8.3 \times 10^{-6}$ & $7.5 \times 10^{-8}$ & $7.7 \times 10^{-6}$ & $1.1 \times 10^{-7}$ & $8.2 \times 10^{-6}$ & $3.5 \times 10^{-5}$ \\
\hline 1500 & $5.5 \times 10^{-5}$ & $7.2 \times 10^{-7}$ & $5.1 \times 10^{-5}$ & $1.2 \times 10^{-6}$ & $5.6 \times 10^{-5}$ & $1.2 \times 10^{-4}$ \\
\hline 2000 & $2.0 \times 10^{-4}$ & $3.9 \times 10^{-6}$ & $1.9 \times 10^{-4}$ & $4.8 \times 10^{-6}$ & $1.6 \times 10^{-4}$ & $2.8 \times 10^{-4}$ \\
\hline 2500 & $5.2 \times 10^{-4}$ & $1.3 \times 10^{-5}$ & $4.9 \times 10^{-4}$ & $1.7 \times 10^{-5}$ & $3.1 \times 10^{-4}$ & $4.8 \times 10^{-4}$ \\
\hline 3000 & $1.0 \times 10^{-3}$ & $3.4 \times 10^{-5}$ & $9.0 \times 10^{-4}$ & $4.5 \times 10^{-5}$ & $5.3 \times 10^{-4}$ & $7.7 \times 10^{-4}$ \\
\hline \multicolumn{7}{|c|}{$\mathrm{HF}-\mathrm{H}_{2}$} \\
\hline 1000 & $\ldots$ & $\ldots$ & $\ldots$ & $\cdots$ & $1.1 \times 10^{-2}$ & $1.6 \times 10^{-2}$ \\
\hline 1500 & $7.9 \times 10^{-5}$ & $\ldots$ & $\ldots$ & $\ldots$ & $1.6 \times 10^{-2}$ & $2.1 \times 10^{-2}$ \\
\hline 2000 & $1.9 \times 10^{-4}$ & $\ldots$ & $\ldots$ & $\ldots$ & $1.9 \times 10^{-2}$ & $2.3 \times 10^{-2}$ \\
\hline 2500 & $3.6 \times 10^{-4}$ & $\ldots$ & $1.6 \times 10^{-3}$ & $2.3 \times 10^{-2}$ & $2.2 \times 10^{-2}$ & $2.6 \times 10^{-2}$ \\
\hline 3000 & $6.1 \times 10^{-4}$ & $2.1 \times 10^{-2}$ & $2.2 \times 10^{-3}$ & $3.3 \times 10^{-2}$ & $2.5 \times 10^{-2}$ & $2.8 \times 10^{-2}$ \\
\hline
\end{tabular}

de Laval nozzle for a hydrogen-fluorine rocket motor may be taken as vibrational near-equilibrium flow. The reactants are taken in such proportion that $\mathrm{H}_{2}$ will be in excess, and the products of combustion passing through the nozzle consist essentially of a mixture of $\mathrm{HF}$ and $\mathrm{H}_{2}$.

Following a procedure analogous to that of Penner in his treatment of chemical reaction in nozzle flow, we take as an estimate for the vibrational temperature lag, $\Delta T$, present for vibrational near-equilibrium flow the relation

$$
\Delta T \cong(-D T / D t) \tau,
$$

where $\tau$ is the vibrational relaxation time, and $D / D t$ is the Euler time derivative. ${ }^{2}$ For representative nozzles in 100 psia thrust motors, it has been found that the cooling rate $(-D T / D t) \cong\left(T_{e}-T_{e}\right) / l_{r} \cong 3 \times 10^{7} \quad{ }^{\circ} \mathrm{K}$ $\mathrm{sec}^{-1} .^{3}$ The use of very small nozzles will increase the rate of cooling, but for nozzles in larger thrust motors $(-D T / D t)$ will be smaller than $3 \times 10^{7} \mathrm{~K} \mathrm{sec}^{-1}$.

From Fig. 2, it can be seen that the probability, $P_{0 \rightarrow 1}(a, b)$, is approximately as large as some of the other probabilities and much larger than the others. The smaller probabilities contribute little to the effective relaxation time. The probabilities which are approximately as large as $P_{0 \rightarrow 1}(a, b)$ all contribute to the effective relaxation time. For the purpose of simplicity, however, let us assume that we may approximate the maximum value of the effective relaxation time as

$$
\tau_{M} \cong 1 / X_{a} X_{b} M_{a b} P_{0 \rightarrow 1}(a, b) .
$$

The actual value of the effective relaxation time must of necessity be smaller than $\tau_{M}$ as the other probabilities, which are approximately as large as $P_{0 \rightarrow 1}(a, b)$, have

2 S. S. Penner, J. Chem. Phys. 19, 877 (1951).

${ }^{3}$ D. Altman and S. S. Penner, J. Chem. Phys. 17, 56 (1949). not been considered in computing $\tau_{M}$ and would lower its value. If we can show, however, that vibrational near-equilibrium flow exists for the calculated upper limit $\tau_{M}$, the conclusion certainly will hold for the real $\tau$ which is smaller than $\tau_{M}$.

In Eq. (12) $X_{a}$ and $X_{b}$ are the mole fractions of $\mathrm{HF}$ and $\mathrm{H}_{2}$ respectively, $M_{a b}$ denotes the number of collisions per second between $\mathrm{HF}$ and $\mathrm{H}_{2}$, and $P_{0 \rightarrow 1}(a, b)$ is the probability that upon a collision an HF molecule will be reduced from the first excited vibrational state to the zeroth state, while the $\mathrm{H}_{2}$ molecule will undergo a change from the zeroth to the first state.

Since $M_{a b}$ increases markedly with pressure it is apparent that if the conditions at the nozzle exit are such that vibrational near-equilibrium flow exists there, then it must also exist everywhere in the nozzle. The value of $\Delta T$ was therefore computed at the nozzle exit where it is a maximum. ${ }^{4}$

In this computation the nozzle exit pressure has been taken as one atmosphere. Also, the product $X_{a} X_{b}$ will vary between the narrow limits of 0.25 and approximately 0.20 and will usually be about 0.23 . The product of $M_{a b}$ and $P_{0 \rightarrow 1}(a, b)$ will not differ appreciably from $4 \times 10^{7} \mathrm{sec}^{-1}$ between $1400^{\circ} \mathrm{K}$ and $3000^{\circ} \mathrm{K}$. Therefore, for exit temperatures between $1400^{\circ} \mathrm{K}$ and $3000^{\circ} \mathrm{K}$ we obtain $\tau_{M} \leq 1 \times 10^{-7} \mathrm{sec}$. For $(-D T / d t) \leq 3 \times 10^{7}{ }^{\circ} \mathrm{K}$ $\sec ^{-1}$, we have $\Delta T_{M} \leq 3^{\circ} \mathrm{K}$.

The estimate that the vibrational temperature lag cannot exceed $3^{\circ} \mathrm{K}$ reasonably indicates that vibrational near-equilibrium flow occurs during expansion in a de Laval nozzle for representative $\mathrm{H}_{2}-\mathrm{F}_{2}$ rocket motors.

We wish to thank Dr. S. S. Penner for helpful discussions.

${ }^{4}$ For a more detailed description of iterative lag calculations during nozzle flow, see S. S. Penner, "Linearization Procedures of the Study of Chemical Reactions in Flow Systems," 1953 Iowa Thermodynamic Symposium, Iowa City (1954). 